\title{
Angiotensin-converting enzyme 2 activator ameliorates severe pulmonary hypertension in a rat model of left pneumonectomy combined with VEGF inhibition
}

\author{
I-Chen Chen ${ }^{1}$, Jao-Yu Lin ${ }^{2}$, Yi-Ching Liu ${ }^{2}$, Chee-Yin Chai ${ }^{3}$, Jwu-Lai Yeh ${ }^{4}$, Jong-Hau \\ $\mathrm{Hsu}^{2}$, Bin-Nan $\mathrm{Wu}^{4}$, and Zen-Kong Dai ${ }^{5}$ \\ ${ }^{1}$ kaohsiung medical university hospital \\ ${ }^{2}$ Kaohsiung Medical University Hospital \\ ${ }^{3}$ Kaohsiung Medical University Chong-Ho memorial Hospital \\ ${ }^{4}$ School of Medicine,Kaohsiung Medical University \\ ${ }^{5}$ Kaohsiung Medical University
}

September 24, 2020

\begin{abstract}
Background Pulmonary artery hypertension (PAH) is a life-threatening and deteriorating disease with no promising therapy available currently due to its diversity and complexity. An imbalance between vasoconstriction and vasodilation has been proposed as the mechanism of PAH. Angiotensin-converting enzyme 2 (ACE2), which catalyzes the hydrolysis of the vasoconstrictor angiotensin (Ang) II into the vasodilator Ang-(1-7), has been shown to be an important regulator of blood pressure and cardiovascular diseases. Herein we hypothesized diminazene aceturate (DIZE), an ACE2 activator, could ameliorate the development of PAH and pulmonary vascular remodeling. Methods A murine model of PAH was established using left pneumonectomy $(\mathrm{PNx})$ on day 0 followed by injection of a single dose of the VEGF receptor-2 inhibitor SU5416 (25mg/kg) subcutaneously on day 1. All hemodynamic and biochemical measurements were done at the end of the study on day 42. Animals were divided into 4 groups ( $\mathrm{n}=6$ /group): (1) sham-operated group, (2) vehicle-treatment group (SuPNx42), (3) early treatment group (SuPNx42/DIZE1-42) with DIZE at $15 \mathrm{mg} / \mathrm{kg} /$ day, subcutaneously from day 1 to day 42, and (4) late treatment group (SuPNx42/DIZE29-42) with DIZE from day 29-42. Results In both the early and late treatment groups, DIZE significantly attenuated the mean pulmonary artery pressure, pulmonary arteriolar remodeling, and right ventricle brain natriuretic peptide (BNP), as well as reversed the overexpression of ACE while up-regulating the expression of Ang-(1-7) when compared with the vehicle-treatment group. In addition, the early treatment group also significantly decreased plasma BNP and increased the expression of eNOS. Conclusions ACE2 activator has therapeutic potentials for preventing and attenuating the development of PAH in an animal model of left pneumonectomy combined with VEGF inhibition. Activation of ACE2 may thus be a useful therapeutic strategy for the treatment of human PAH.
\end{abstract}

\section{Angiotensin-converting enzyme 2 activator ameliorates severe pulmonary hypertension in a rat model of left pneumonectomy combined with VEGF inhibition}

I-Chen Chen, MD ${ }^{1,2,3}$, Jao-Yu Lin, MD ${ }^{4}$, Yi-Ching Liu, MD ${ }^{1}$, Chee-Yin Chai, MD, PhD ${ }^{5}$, Jwu-Lai Yeh, $\mathrm{PhD}^{3,6}$, Jong-Hau Hsu, MD, PhD, ${ }^{1,2}$, Bin-Nan Wu, $\mathrm{PhD}^{3,6}$ and Zen-Kong Dai, MD, PhD,,$^{1,2,3^{*}}$

${ }^{1}$ Department of Pediatrics, Kaohsiung Medical University Hospital, Kaohsiung, Taiwan

${ }^{2}$ Department of Pediatrics, School of Medicine, College of Medicine, Kaohsiung Medical University, Kaohsiung, Taiwan 
${ }^{3}$ Graduate Institute of Medicine, College of Medicine, Kaohsiung Medical University, Kaohsiung, Taiwan

${ }^{4}$ Department of Surgery, Kaohsiung Medical University, Kaohsiung, Taiwan

${ }^{5}$ Department of Pathology, School of Medicine, College of Medicine, Kaohsiung Medical University, Kaohsiung, Taiwan

${ }^{6}$ Department of Pharmacology, School of Medicine, College of Medicine, Kaohsiung Medical University, Kaohsiung, Taiwan

Correspondence: Zen-Kong Dai

Zen-Kong Dai, MD, PHD

Department of Pediatrics, Kaohsiung Medical University, 100 Shih-Chuan 1st Road, Sun-Ming District, Kaohsiung 807, Taiwan

Tel: 886-7-3121101-6507 E-mail: zenkong@kmu.edu.tw Fax: 886-7-3208316

Running title: ACE2 activator ameliorates pulmonary artery hypertension

\section{Summary}

\section{Background}

Pulmonary artery hypertension (PAH) is a life-threatening and deteriorating disease with no promising therapy available currently due to its diversity and complexity. An imbalance between vasoconstriction and vasodilation has been proposed as the mechanism of PAH. Angiotensin-converting enzyme 2 (ACE2), which catalyzes the hydrolysis of the vasoconstrictor angiotensin (Ang) II into the vasodilator Ang-(1-7), has been shown to be an important regulator of blood pressure and cardiovascular diseases. Herein we hypothesized diminazene aceturate (DIZE), an ACE2 activator, could ameliorate the development of PAH and pulmonary vascular remodeling.

\section{Methods}

A murine model of PAH was established using left pneumonectomy (PNx) on day 0 followed by injection of a single dose of the VEGF receptor-2 inhibitor SU5416 $(25 \mathrm{mg} / \mathrm{kg})$ subcutaneously on day 1 . All hemodynamic and biochemical measurements were done at the end of the study on day 42 . Animals were divided into 4 groups ( $\mathrm{n}=6$ /group): (1) sham-operated group, (2) vehicle-treatment group $\left(\mathrm{SuPNx}_{42}\right)$, (3) early treatment group $\left(\mathrm{SuPNx}_{42} / \mathrm{DIZE}_{1-42}\right)$ with DIZE at $15 \mathrm{mg} / \mathrm{kg} /$ day, subcutaneously from day 1 to day 42 , and (4) late treatment group $\left(\mathrm{SuPNx}_{42} / \mathrm{DIZE}_{29-42}\right)$ with DIZE from day 29-42.

Results In both the early and late treatment groups, DIZE significantly attenuated the mean pulmonary artery pressure, pulmonary arteriolar remodeling, and right ventricle brain natriuretic peptide( BNP), as well as reversed the overexpression of ACE while up-regulating the expression of Ang-(1-7) when compared with the vehicle-treatment group. In addition, the early treatment group also significantly decreased plasma $\mathrm{BNP}$ and increased the expression of eNOS.

Conclusions ACE2 activator has therapeutic potentials for preventing and attenuating the development of PAH in an animal model of left pneumonectomy combined with VEGF inhibition. Activation of ACE2 may thus be a useful therapeutic strategy for the treatment of human PAH.

Key words: pulmonary artery hypertension; left pneumonectomy; SU5416; ACE2 activator; diminazene aceturate; ACE2-Ang (1-7)-Mas axis

\section{Introduction}

Pulmonary hypertension $(\mathrm{PH})$ is a progressive and presently incurable disorder, which complicates the majority of cardiovascular and respiratory diseases. ${ }^{1,2}$ The etiology of $\mathrm{PH}$ is classified by WHO into the following five distinct groups: (1) pulmonary artery hypertension (PAH), (2) PH due to left heart disease, (3) PH 
due to lung disease and/or hypoxia, (4) chronic thromboembolic pulmonary hypertension, and (5) unclear multifocal mechanisms. ${ }^{3}$ All of these groups share a mean, resting, pulmonary arterial pressure (PAP) of greater than $20 \mathrm{mmHg} .{ }^{4}$ The pathogenesis of $\mathrm{PH}$ is complexed and multifactorial; proliferative vasculopathy, pulmonary vascular remodeling, vascular constriction, and endothelial cell dysfunction have been proven in several previous studies. ${ }^{5,6}$

As rodent models are beneficial for understanding the pathophysiology of $\mathrm{PH}$ and for testing experimental therapies, more and more rodent models have been developed and many of them have exhibited characteristics mimicking WHO's classification of human $\mathrm{PH}^{6}$ Traditionally, rat models of pulmonary artery hypertension (PAH) include monocrotaline (with or without pneumonectomy and/or hypoxia), chronic hypoxia together with SU5416 (3-(3,5-dimethyl-1H-pyrrol-2-ylmethylene)-1,3-dihydroindol-2-one; a VEGF (Vascular endothelial growth factor) receptor-2 inhibitor, and the fawn-hooded rat (FHR). ${ }^{7-9}$ A major advantage of the SU5416 hypoxia models over that of the monocrotaline hypoxia one is the remodeling of neointimal occlusive lesion, which accumulates unique tumorlets of endothelial cells and obliterates medium-sized precapillary arteries. It has also been shown that the SU5416 hypoxia model developed pulmonary arterial changes resembling plexiform-like lesions, which are characteristic features of human PAH, by increasing apoptosis of endothelial cells followed by converting them into apoptosis resistant and phenotypically altered endothelial cells. ${ }^{10}$ Recently, a newly developed rodent PAH model that combined left pneumonectomy with SU5416 was reported. ${ }^{11}$ Unlike the traditional SU5416 hypoxia model in which a partial reversal of PAH is seen upon returning to normoxia, this new animal model does not reverse hypoxia-induced vasoconstriction and hemoconcentration under similar conditions. Rather, the right ventricular systolic pressure increased gradually over time, a feature that may favor the assessment of drug effects in preclinical trials. ${ }^{11}$

The Renin-angiotensin system (RAS) has been implicated in playing a causative role in PH. ${ }^{12,13}$ There are two opposing arms in RAS: the presser arm, composed of angiotensin converting enzyme (ACE), angiotensin II (Ang II) produced from Ang I by ACE, and the Ang II type 1 (AT1) receptor as the main protein mediating the biological actions of Ang II; and the vasodilator arm, consisting angiotensin converting enzyme 2 (ACE2), Ang-(1-7) generated through hydrolysis of Ang II by ACE2, and the Mas receptor as the protein conveying the vasodilatory, antiproliferative, antifibrotic, and antithrombotic effects of Ang-(1-7). ${ }^{12,13,14}$ Therefore, an increase in the levels of Ang-(1-7) should have beneficial effects for PAH. However, targeting the ACE2/Ang (1-7)/Mas receptor pathway in PAH is still under scrutiny.

ACE2 has exhibited its modulating ability in the balance between vasoconstriction and vasodilation in many experiments. ${ }^{15,16}$ Recently, diminazene aceturate (DIZE), a Food and Drug Administration (FDA) approved anti-trypanosomal drug, has been demonstrated to exert off-target effect on activating ACE2 ${ }^{17,18}$ This effect of DIZE has been confirmed through the analysis of cleavage of the vasoconstrictor peptide Ang II, the most physiologically relevant natural substrate for ACE2. ${ }^{17}$

On the basis of the benefits demonstrated by DIZE in other PH models, ${ }^{18-20}$ we aimed to evaluate its effects on a rat model of PAH induced by left pneumonectomy combined with SU5416. We reasoned that in this model there could be an up-regulated expression of ACE, leading to the imbalance between vasoconstriction and vasodilation. Treatment with DIZE would increase the levels of ACE2, and hence, the vasodilatory peptide Ang-(1-7) to prevent and ameliorate the development of PAH.

\section{Methods}

\section{Animal Model of PAH}

All protocols had been approved by the animal research committee of Kaohsiung Medical University (IACUC approval NO: 104226). Male Wistar rats weighing approximately $220 \mathrm{~g}$ were purchased from National Animal Center, Taiwan. after arriving at the Kaohsiung Medical University vivarium, the rats were acclimated for at least one week before being used in the experiment. They were housed in a room on a 12-hr light/dark cycle under controlled temperature of 22.1 and relative humidity of $55 \%$, and they were provided with normal chow and water ad libitum. On the day of experiment, animals were anesthetized with a mixture of sodium pentobarbitone $(20 \mathrm{mg} / \mathrm{kg}$, i.p. $)$ and ketamine $(40 \mathrm{mg} / \mathrm{kg}$, i.m.) under orotracheally ventilated 
rodent respirators (Harvard, South Natick, MA) and lateral thoracotomy in the third intercostal space was performed. Left lung of rats was resected and closed by clip ligation (Ligaclip multiple clip applier, medium size, Ethicon Endo-Surgery), and the animals were suture to prevent pneumothorax. The chest retractor was then removed after 15 minutes, and the thymus was moved back to its physiological position. The chest cavity and skin were closed. The entire procedure took less than $30 \mathrm{~min}$ for each rat. After operation, rats were extubated and received an additional injection of sterile saline $(10 \mathrm{ml})$. On the following day, SU5416 $(25 \mathrm{mg} / \mathrm{kg})$ was injected subcutaneously in surviving rats. Sham-operated animals underwent identical operation without pneumonectomy $(\mathrm{PNx})$ and received sterile saline $(20 \mathrm{mg} / \mathrm{kg})$ subcutaneously, instead. The day of SU5416 injection was designated as day 0. All animals were individually housed in a 12-h dark/light cycle-controlled room and fed a regular rat diet. The procedure was also shown in supplemental figure 1.

\section{Study design}

All rats were randomized ( $\mathrm{n}=6$ / group) to undergo the sham operation (group 1) or SU5416 injection with left pneumonectomy (SuPNx). The SuPNx rats were further randomized for subcutaneous treatment with saline $\left(\mathrm{SuPNx}_{42}\right)$ (group 2) or DIZE (Santa Cruz Biotechnology, CA, USA) at a dose of $15 \mathrm{mg} / \mathrm{kg} /$ day from day 1 to day $42\left(\mathrm{SuPNx}_{42} / \mathrm{DIZE}_{1-42}\right)$ in the early treatment protocol (group 3) or from day 29 to day $42\left(\mathrm{SuPNx}_{42} / \mathrm{DIZE}_{29-42}\right)$ in the late treatment protocol (group 4$)$. On day 42 , all rats were ventilated and sacrificed after checking hemodynamic data. The lungs and heart from each rat were rapidly perfused with sterile saline under a pressure of $100-\mathrm{cm} \mathrm{H}_{2} \mathrm{O}$ prior to removal. One-half of the lung tissue was homogenized for protein extraction, and the other half was kept in $10 \%$ formaldehyde and froze in $4^{\circ} \mathrm{C}$ for histopathologic analysis.

\section{Hemodynamics Measurements}

The method was described in our previous published study. ${ }^{21,22}$ Briefly, a PE-50 catheter (Beckton-Dickinson, Sparks, MD, USA) was inserted into the femoral artery using a cut-down procedure for recording pressure. Thereafter, a left parasternal thoracotomy was performed. Another catheter was inserted closely to the main pulmonary artery via the right ventricular outflow tract. Pulmonary and femoral arterial pressures were recorded simultaneously.

\section{Plasma and right ventricle brain natriuretic peptide (BNP)}

Plasma samples $(1.0 \mathrm{~mL})$ and homogenous right ventricle (RV) were expressed in v/v for TFA and homogenate in $\mathrm{w} / \mathrm{v}$ and then centrifuged at $2000 \times \mathrm{g}$ for $15 \mathrm{~min}$ at $4^{\circ} \mathrm{C}$. Enzyme-immunoassay kits (Phoenix Pharmaceuticals, Burlingame, CA, USA) were used to measure plasma and RV BNP contents according to the manufactural protocol.

\section{Histopathologic and immunohistochemical Analysis}

Lung tissues which were immersed in formalin for at least $24 \mathrm{hr}$ were analyzed for histopathology. Hematoxylin-Eosin (H-E) staining was used for analysis of the pulmonary arterioles in terms of medial wall thickness under a microscope at a magnification of 400X. The methods were described in our previous published study. ${ }^{21,22}$ and the medial wall thickness of each arteriole was expressed as follows:

Percent wall thickness $=($ medial thickness $\times 2$ /external diameter $) \times 100$.

Verhoeff-van Gieson staining was performed for evaluating the severity of neointimal formation using a modified scoring system published before. ${ }^{23-25}$ The degree of vascular remodeling was determined by measuring 30 transversally cut vessels (outer diameter: 50-100 $\mu \mathrm{m}$ ) from each rat and expressing the thickness of the combined intimal and medial layers as a percentage of total vessel diameter. The percentage of vascular occlusion of these 30 vessels was categorized as Grade 0: no evidence of luminal obstruction; Grade 1: the presence of partial $(<50 \%)$ luminal occlusion; and Grade 2: the present of luminal occlusion greater than $50 \%$. An average Grade score for 30 vessels was calculated for each animal. Afterwards, a quantitative 
analysis of luminal obstruction in 30 consecutive small pulmonary arteries from all rats in each group was performed.

In addition, double immunohistochemical staining was performed to present the smooth muscle actin (SMA) by using formalin-fixed, paraffin-embedded tissue. Experiments were performed by double staining polymer detection systems (TADS03, BioTnA, Taiwan) using rabbit polyclonal anti-SMA (1:100, Cell signaling, MA, USA) and anti-PCNA (Proliferating Cell Nuclear Antigen)(1:100, Cell signaling, MA, USA) antibodies.

\section{Western blot analysis of pulmonary eNOS, ACE, ACE2, and MAS}

Lung tissues $(100 \mathrm{mg})$ were homogenized in $1 \mathrm{ml}$ of RIPA buffer (1\% Triton X-100, $15 \mathrm{mM}$ HEPES-NaOH [pH 7.5], $0.15 \mathrm{mM} \mathrm{NaCl}, 1 \%$ sodium deoxycholate, $0.1 \%$ SDS, $1 \mathrm{mM}$ sodium orthovanadate, $10 \mathrm{mM}$ EDTA, and $0.5 \%$ protease inhibitor cocktail [Sigma, St. Louis, MO, USA]) and centrifuged at $15,000 \times \mathrm{g}$ for 20 $\min$ at $4^{\circ} \mathrm{C}$. Proteins in the supernatant $(100 \mu \mathrm{g})$ were subjected to SDS-PAGE in $10 \%$ polyacrylamide gels and were transferred onto PVDF membranes (Pall, Port Washington, NY, USA). Each membrane was blocked with $5 \%$ non-fat dry milk in Tris-buffered saline (TBS), probed with anti-actin $(1: 10,000)$ (Upstate Biotechnology, Lake Placid, NY, USA), anti-eNOS ( 1:1000, Millipore, Milford, MA, USA) anti-ACE, antiACE2 (1:500 dilution; Millipore, Billerica, MA, USA), or anti-MAS antibodies (1:1,000 dilution; Millipore), and then incubated with horseradish peroxidase-conjugated secondary antibody. (Leadgene Biomedical, TW) Signals were detected using the Western Lighting@ chemiluminescent kit (Millipore) according to the manufacturer's specifications.

\section{Ang II and Ang-(1-7) measurements}

RV and lung supernatants were acidified with $0.6 \%$ trifluoroacetic acid (TFA) to obtain a $10 \%$ (w/v) homogenate. The samples were centrifuged at $2,000 \times \mathrm{g}$ for $15 \mathrm{~min}$ at $4^{\circ} \mathrm{C}$, and dried under a steam of nitrogen at $60^{\circ} \mathrm{C}$. Enzyme-immunoassay kits (Phoenix Pharmaceuticals, CA, USA) were used to measure the Ang II and Ang-(1-7) contents in the RV and lung.

\section{Statistical analysis}

The results obtained from ELISA and Western blots were analyzed by densitometry and expressed as means \pm standard error of the mean. All data from the four groups were analyzed using one-way analysis of variance (ANOVA) followed by Tukey's test. Comparison of the beneficial efficacy of DIZE between the early treatment and the late treatment protocols was conducted by two-way ANOVA ( $\mathrm{n}=6$ rats/group). A P value $<0.05$ was considered statistically significant.

\section{Results}

\section{DIZE reduced RV systolic pressure and RV and plasma BNP}

Significant increases in the right ventricular systolic pressure (RVSP) were observed in the $\mathrm{SuPNx}_{42}$ groups when compared with the sham-operated rats (Figure $2 \mathrm{~A})$. Both early $\left(\mathrm{SuPNx}_{42} / \mathrm{DIZE}_{1-42}\right)$ and late DIZE treatment $\left(\mathrm{SuPNx}_{42} / \mathrm{DIZe}_{29-42}\right)$ groups decreased the RVSP when compared with the vehicle treatment group (Figure 2A). The Fulton index, weight ratio of right ventricle to left ventricle plus septum (RV/LV+S), in the SuPNx42 rats was significantly increased than the sham-operated rats, which indicated the right ventricle was hypertrophied in the SuPNx42 animals (Figure 2B). However, the RV/LV+S ratio in either the early or late DIZE treatment group was not different when compared to $\mathrm{SuPNx}_{42}$ group (Figure 2B). There were no significant changes among all 4 groups in the lung or body weight (Figures 2C and 2D).

Plasma BNP, an index of heart failure, was elevated significantly in the $\mathrm{SuPNx}_{42}$ group compared with the sham-operated rats. In the early treatment group $\left(\mathrm{SuPNx}_{42} / \mathrm{DIZE}_{1-42}\right)$, DIZE resulted in significant decreases in plasma BNP compared to that of vehicle-treated $\mathrm{SuPNx}_{42}$ rats, but no significant changes were seen in the late treatment group ( $\left.\mathrm{SuPNx}_{42} / \mathrm{DIZE}_{29-42}\right)$ (Figure $\left.3 \mathrm{~A}\right)$.

Similar to the plasma BNP, RV BNP was also elevated significantly in $\mathrm{SuPNx}_{42}$ group compared with the sham-operated rats. Both the early treatment group $\left(\mathrm{SuPNx}_{42} / \mathrm{DIZE}_{1-42}\right)$ and the late treatment group 
$\left(\mathrm{SuPNx}_{42} / \mathrm{DIZE}_{29-42}\right)$ displayed significant decreases in the levels of RV BNP compared to that found in the vehicle-treated $\mathrm{SuPNx}_{42}$ rats (Figure 3B).

DIZE attenuated pulmonary vascular remodeling and smooth muscle neointimal proliferation

Using H-E staining, the medial wall thickness of pulmonary arterioles (50-100 $\mu \mathrm{m}$ in diameter) was found to increase significantly in the $\mathrm{SuPNX}_{42}$ rats compared with that in sham-operated rats (Figures 4A and 4D). Early DIZE treatment $\left(\mathrm{SuPNx}_{42} / \mathrm{DIZE}_{1-42}\right)$ significantly attenuated the medial wall thickness of pulmonary arterioles, when compared with the vehicle-treated rats $\left(\mathrm{SuPNx}_{4}\right)$ (Figures $4 \mathrm{~A}$ and $\left.4 \mathrm{D}\right)$. However, no significant changes in medial wall thickness were observed in the $\mathrm{SuPNx}_{42} / \mathrm{DIZE}_{29-42}$ group when compared with that of the $\mathrm{SuPNx}_{42}$ rats.

In addition, Verhoeff-van Gieson staining was carried out for evaluating the severity of neointimal formation (Figure 4B). The distribution of vascular lesions and an average vascular occlusion score (VOS) are presented in Figure 4E. The $\mathrm{SuPNx}_{42}$ rats developed severe pulmonary vascular remodeling with a VOS of $1.27 \pm 0.74$. In the early treatment group with DIZE, the VOS was significant decreased (0.87 \pm 0.54$)$ compared to the $\mathrm{SuPNx}_{42}$ rats $(p<0.05)$. There was also a trend of decrease in the late treatment group with a VOS of 1.07 \pm 0.79 when compared to the $\mathrm{SuPNx}_{42}$ rats.

\section{DIZE Regulated the expressions of eNOS, ACE, and ACE2/MAS Axis in SuPNx Rats}

Figure 5A shows a representative pulmonary expression of eNOS, ACE, ACE2, MAS by Western blot analysis. $\mathrm{SuPNX}_{42}$ has the trend to upregulated pulmonary eNOS expression when compared with the sham-operated rats. Pulmonary expression of eNOS was significantly elevated in the early treatment protocol when compared with the $\mathrm{SuPNx}_{42}$ groups, but this was not seen in the late treatment protocol (Figure 5B).

Compared to the sham-operated rats, the ratio of pulmonary expression of ACE was elevated in the $\mathrm{SuPNx}_{42}$ group. Significant decreases in ACE protein expression in both early and late DIZE treatment groups were noted when compared with the vehicle-treated $\mathrm{SuPNx}_{42}$ rats. (Figure $5 \mathrm{C}$ )

Pulmonary expression of ACE2 was increased in the early treatment group ( $\left.\mathrm{SuPNX}_{42} / \mathrm{DIZE}_{1-42}\right)$ compared to the $\mathrm{SuPNx}_{42}$ rats. No significant differences were found among the sham $42, \mathrm{SuPNx}_{42}$, and $\mathrm{SuPNX}_{42} / \mathrm{DIZE}_{29-42}$ groups (Figure 5D).

The Mas is a G protein-coupled receptor with its vasoprotective effect in RAS system. ${ }^{14}$ In western blot analysis, there were no significant changes in Mas expression among all four groups. (Figure 5E)

\section{DIZE upregulated RV and pulmonary Ang-(1-7) levels}

The RV and pulmonary tissue Ang-(1-7) levels were not changed in the $\mathrm{SuPNx}_{42}$ group when compared to the sham-operated rats (Figures $6 \mathrm{~A}$ and $6 \mathrm{~B}$ ). However, there was a significant increase of RV Ang-(1-7) in both the early and late DIZE treatment groups when compared with the vehicle-treated $\mathrm{SuPNx}_{42}$ rats (Figures 6A and 6B). In addition, early DIZE treatment also increased the levels of pulmonary Ang-(17) when compared with the $\mathrm{SuPNx}_{42}$ group (Figure $6 \mathrm{~B}$ ). No significant differences were found among the sham $42, \mathrm{SuPNx}_{42}$, and $\mathrm{SuPNx}_{42} / \mathrm{DIZE}_{29-42}$ groups (Figure $6 \mathrm{~B}$ ).

\section{Discussion}

SU5416 was the first VEGF receptor 2 inhibitor to enter clinical development for cancer therapy. ${ }^{26}$ It has been shown that inhibiting the VEGF- and endothelial-dependent proliferation will result in structural changes known as plexiform lesions. ${ }^{10,26-29}$ Allowing for the selection of an apoptosis resistant, proliferating endothelial cell phenotype by the combination of blockade of VEGF receptor 2 and hypoxia, severe PAH will develop. ${ }^{26}$ In this study, we reasoned that a pulmonary insult such as pneumonectomy followed by injection with SU5416 will yield similar results. In fact, our SuPNx model was shown to cause severe angio-obliterative PAH associated with increased cell proliferation and proapoptotic signaling, resulting in neointimal and medial remodeling. ${ }^{11}$ In addition, unlike the hypoxia model where partial reversibility of 
pulmonary hypertension is seen after returning to normoxia, the SuPNx model is independent of hypoxic vasoconstriction and hemoconcentration. ${ }^{11}$

In our study, the RVSP in the SUPNx 42 rats increased significantly, which may favor the assessment of drug effects in preclinical trials. The RVSP improved significantly in both the early and late DIZE treatment protocols, suggesting the effectiveness of hemodynamic changes by DIZE in PAH. However, although the Fulton index did increase in the $\mathrm{SuPNx}_{42}$ rats, there were only trends of improvement with DIZE in either the early or the late treatment protocols. It is possible that, due to the severe pathologic changes in this chronic PAH model, the observed hypertrophy in cardiomyocytes was irreversible, leading to heart failure.

In addition to endothelin, RAS has also been implicated as a causative factor in PAH. ${ }^{1}$ Ang II, a principal effector peptide of the RAS, can exert deleterious effects on the pulmonary vasculature resulting in vasoconstriction, proliferation, and inflammation, all of which are contributable to the development of PAH. However, it is difficult to measure the plasma and tissue levels of Ang II due to its very short half-life (16 \pm $1 \mathrm{~s}$ in mice) ${ }^{30}$ In contrast, ACE, which catalyzes the conversion of Ang I to Ang II, is abundant in the small pulmonary arteries and is therefore more easily to be detected ${ }^{31}$. Thus, measuring ACE, instead of Ang II levels in the lung, provides a more practical method for assessing the associated hemodynamic changes. In our study, there were no significant changes in pulmonary Ang II levels among the four animal groups. However, the expression of pulmonary ACE was increased in $\mathrm{SuPNx}_{42}$ rats and ameliorated by DIZE in both early and late treatment group, suggesting that ACE may be a representative marker in in this animal model of PAH.

In the RAS, ACE/Ang II/AT1 constitutes the vasopressor arm, which is counterbalanced by the ACE2/Ang(1-7)/Mas receptor axis. ${ }^{32}$ By converting Ang II to the vasodilatory peptide Ang-(1-7), ACE2 provides a negative feedback on the RAS and protects the major organs such as heart and kidneys from being damaged by excessive Ang II generated during the development of PAH. ${ }^{33,34}$ Interestingly, in the present study, the RV and pulmonary levels of Ang-(1-7) were not significantly altered in the $\mathrm{SuPNx}_{42}$ group when compared with the sham-operated rats. However, the RV levels of Ang-(1-7) were significantly elevated in both the early and late DIZE treatment groups, and the pulmonary levels of this peptide were also significantly increased in the early DIZE treatment group.

It should be noted that, besides the action of ACE2, Ang-(1-7) can also be formed by other biochemical pathways. It can be generated from hydrolysis of angiotensin I by neprilysins (NEPs) or cleavage of the Ang(1-9) by ACE. ${ }^{35-38}$ With respect to its metabolism, Ang-(1-7) can be subsequently degraded by ACE to form Ang-(1-5), by dipeptidyl peptidase 3 (DPP3) to produce Ang-(3-7) and Ang-(5-7), or by aminopeptidase A (APA) to generate Ang-(2-7). ${ }^{38}$ Affecting the activity of any of the aforementioned enzymes will undoubtedly result in a change of the levels of Ang-(1-7). To this end, it is speculated other alternative pathways may also influence the formation of Ang-(1-7) in our animal model. Further studies are needed to fully elucidate the involved biochemical pathways.

It has been reported that Ang-(1-7) can stimulate the releases of endothelial derived nitric oxide (eNOS) and vasodilator prostaglandins as well as potentiate the vasodilatory effect of bradykinin. ${ }^{39-41}$ Consistent with these results, eNOS was significantly elevated in our early DIZE treatment group when compared to $\mathrm{SuPNx}_{42}$ rats (Figure 5B), inferring that Ang-(1-7) may stimulate eNOS release in this animal model. The increased expression of eNOS could contribute to the lowering of pulmonary artery pressure observed herein (Figure 2A).

In summary, our model of hypertensive pulmonary vascular disease in pneumonectomized, SU5416-injected rats resemble the neointimal proliferation and vascular occlusion by smooth muscle cells that occurs in human $\mathrm{PAH}$. The efficacy of DIZE in the early and late treatment group suggests its ability to rescue animals from established hypertensive pulmonary vascular disease. However, the exact mechanisms by which DIZE exerts its beneficial effects remain to be investigated. From our results, it is likely that attenuation of PAH by DIZE involves a combination of antiproliferative effects on pulmonary vascular smooth muscle cells through production of Ang-(1-7), suppression of the growth of vascular smooth muscle cells, and also induction of 
endothelial cell eNOS expression. ${ }^{41,42}$

\section{Conclusion}

The present rat model of PAH showed elevated ACE protein levels in the lung as well as histopathological findings such as Increased intimal and medial fractions accompanied by obliterative lesions. DIZE, an ACE2 activator, reduced RVSP, medial wall thickness, and intimal fraction in pulmonary arterioles. In addition, it upregulates pulmonary expression of the ACE2/Ang-(1-7)/Mas receptor axis, leading to a therapeutic effect in this rat model of PAH. Our study showed that DIZE may be a potential agent for the treatment of PAH.

\section{Competing interests}

The authors state no conflict of interest.

\section{Acknowledgments}

This study was supported by grants from Ministry of Science and Technology, R.O.C (MOST 106-2314B-037 -078 and 105-2314-B-037 -011) and Kaohsiung Medical University Hospital (KMUH105-5R36 and KMUH106-6R43).

The authors thank Mr. Chao-Hsiung Chen and Chia-An Yeh for technical support.

\section{References}

1. Mercurio V, Bianco A, Campi G, Cuomo A, Diab N, Mancini A, Parrella P, Petretta M, Hassoun PM, Bonaduce D. New Drugs, Therapeutic Strategies, and Future Direction for the Treatment of Pulmonary Arterial Hypertension. Curr Med Chem.2019;26(16):2844-2864.

2. Galie N, Humbert M, Vachiery JL, Gibbs S, Lang I, Torbicki A, Simonneau G, Peacock A, Vonk Noordegraaf A, Beghetti M, et al. 2015 ESC/ERS Guidelines for the diagnosis and treatment of pulmonary hypertension: The Joint Task Force for the Diagnosis and Treatment of Pulmonary Hypertension of the European Society of Cardiology (ESC) and the European Respiratory Society (ERS): Endorsed by: Association for European Paediatric and Congenital Cardiology (AEPC), International Society for Heart and Lung Transplantation (ISHLT). Eur Respir J. 2015;46(4):903-975.

3. Simonneau G, Gatzoulis MA, Adatia I, Celermajer D, Denton C, Ghofrani A, Gomez Sanchez MA, Krishna Kumar R, Landzberg M, Machado RF, et al. Updated clinical classification of pulmonary hypertension. $J$ Am Coll Cardiol. 2013;62(25 Suppl):D34-41.

4. Simonneau G, Montani D, Celermajer DS, Denton CP, Gatzoulis MA, Krowka M, Williams PG, Souza R. Haemodynamic definitions and updated clinical classification of pulmonary hypertension. Eur Respir J. 2019;53(1).

5. Pietra GG, Capron F, Stewart S, Leone O, Humbert M, Robbins IM, Reid LM, Tuder RM. Pathologic assessment of vasculopathies in pulmonary hypertension. J Am Coll Cardiol.2004;43(12 Suppl S):25S-32S.

6. Leopold JA, Maron BA. Molecular Mechanisms of Pulmonary Vascular Remodeling in Pulmonary Arterial Hypertension. Int J Mol Sci. 2016;17(5).

7. Ciuclan L, Bonneau O, Hussey M, Duggan N, Holmes AM, Good R, Stringer R, Jones P, Morrell NW, Jarai G, et al. A novel murine model of severe pulmonary arterial hypertension. Am J Respir Crit Care Med. 2011;184(10):1171-1182.

8. Ryan JJ, Marsboom G, Archer SL. Rodent models of group 1 pulmonary hypertension. Handb Exp Pharmacol. 2013;218:105-149.

9. Ryan J, Bloch K, Archer SL. Rodent models of pulmonary hypertension: harmonisation with the world health organisation's categorisation of human PH. Int J Clin Pract Suppl. 2011(172):15-34. 
10. Sakao S, Tatsumi K. The effects of antiangiogenic compound SU5416 in a rat model of pulmonary arterial hypertension. Respiration. 2011;81(3):253-261.

11. Happe CM, de Raaf MA, Rol N, Schalij I, Vonk-Noordegraaf A, Westerhof N, Voelkel NF, de Man FS, Bogaard HJ. Pneumonectomy combined with SU5416 induces severe pulmonary hypertension in rats. Am J Physiol Lung Cell Mol Physiol.2016;310(11):L1088-1097.

12. Tan WSD, Liao W, Zhou S, Mei D, Wong WF. Targeting the renin-angiotensin system as novel therapeutic strategy for pulmonary diseases. Curr Opin Pharmacol.2017;40:9-17.

13. Ferreira AJ, Shenoy V, Yamazato Y, Sriramula S, Francis J, Yuan L, Castellano RK, Ostrov DA, Oh SP, Katovich MJ, et al. Evidence for angiotensin-converting enzyme 2 as a therapeutic target for the prevention of pulmonary hypertension.Am J Respir Crit Care Med. 2009;179(11):1048-1054.

14. Povlsen AL, Grimm D, Wehland M, Infanger M, Kruger M. The Vasoactive Mas Receptor in Essential Hypertension. J Clin Med. 2020;9(1).

15. Burrell LM, Johnston CI, Tikellis C, Cooper ME. ACE2, a new regulator of the renin-angiotensin system. Trends Endocrinol Metab. 2004;15(4):166-169.

16. Velkoska E, Patel SK, Burrell LM. Angiotensin converting enzyme 2 and diminazene: role in cardiovascular and blood pressure regulation. Curr Opin Nephrol Hypertens.2016;25(5):384-395.

17. Kulemina LV, Ostrov DA. Prediction of off-target effects on angiotensin-converting enzyme 2.J Biomol Screen. 2011;16(8):878-885.

18. Shenoy V, Gjymishka A, Jarajapu YP, Qi Y, Afzal A, Rigatto K, Ferreira AJ, Fraga-Silva RA, Kearns $\mathrm{P}$, Douglas JY, et al. Diminazene attenuates pulmonary hypertension and improves angiogenic progenitor cell functions in experimental models. Am J Respir Crit Care Med. 2013;187(6):648-657.

19. Li G, Liu Y, Zhu Y, Liu A, Xu Y, Li X, Li Z, Su J, Sun L. ACE2 activation confers endothelial protection and attenuates neointimal lesions in prevention of severe pulmonary arterial hypertension in rats. Lung. 2013;191(4):327-336.

20. Haga S, Tsuchiya H, Hirai T, Hamano T, Mimori A, Ishizaka Y. A novel ACE2 activator reduces monocrotaline-induced pulmonary hypertension by suppressing the JAK/STAT and TGF-beta cascades with restored caveolin-1 expression. Exp Lung Res. 2015;41(1):21-31.

21. Chen IC, Tan MS, Wu BN, Chai CY, Yeh JL, Chou SH, Chen IJ, Dai ZK. Statins ameliorate pulmonary hypertension secondary to left ventricular dysfunction through the Rho-kinase pathway and NADPH oxidase. Pediatr Pulmonol.2017;52(4):443-457.

22. Dai ZK, Wu BN, Chen IC, Chai CY, Wu JR, Chou SH, Yeh JL, Chen IJ, Tan MS. Attenuation of pulmonary hypertension secondary to left ventricular dysfunction in the rat by Rho-kinase inhibitor fasudil. Pediatr Pulmonol. 2011;46(1):45-59.

23. Faul JL, Nishimura T, Berry GJ, Benson GV, Pearl RG, Kao PN. Triptolide attenuates pulmonary arterial hypertension and neointimal formation in rats. Am J Respir Crit Care Med. 2000;162(6):2252-2258.

24. Nishimura T, Faul JL, Berry GJ, Vaszar LT, Qiu D, Pearl RG, Kao PN. Simvastatin attenuates smooth muscle neointimal proliferation and pulmonary hypertension in rats. Am J Respir Crit Care Med. 2002;166(10):1403-1408.

25. Nishimura T, Vaszar LT, Faul JL, Zhao G, Berry GJ, Shi L, Qiu D, Benson G, Pearl RG, Kao PN. Simvastatin rescues rats from fatal pulmonary hypertension by inducing apoptosis of neointimal smooth muscle cells. Circulation.2003;108(13):1640-1645.

26. Taraseviciene-Stewart L, Kasahara Y, Alger L, Hirth P, Mc Mahon G, Waltenberger J, Voelkel NF, Tuder RM. Inhibition of the VEGF receptor 2 combined with chronic hypoxia causes cell death-dependent 
pulmonary endothelial cell proliferation and severe pulmonary hypertension. FASEB J. 2001;15(2):427-438.

27. Folkman J. Angiogenesis research: from laboratory to clinic. Forum (Genova). 1999;9(3 Suppl 3):59-62.

28. Fong TA, Shawver LK, Sun L, Tang C, App H, Powell TJ, Kim YH, Schreck R, Wang X, Risau W, et al. SU5416 is a potent and selective inhibitor of the vascular endothelial growth factor receptor (Flk-1/KDR) that inhibits tyrosine kinase catalysis, tumor vascularization, and growth of multiple tumor types. Cancer Res. 1999;59(1):99-106.

29. Mendel DB, Laird AD, Smolich BD, Blake RA, Liang C, Hannah AL, Shaheen RM, Ellis LM, Weitman S, Shawver LK, et al. Development of SU5416, a selective small molecule inhibitor of VEGF receptor tyrosine kinase activity, as an anti-angiogenesis agent. Anticancer Drug Des. 2000;15(1):29-41.

30. Al-Merani SA, Brooks DP, Chapman BJ, Munday KA. The half-lives of angiotensin II, angiotensin IIamide, angiotensin III, Sar1-Ala8-angiotensin II and renin in the circulatory system of the rat. $J$ Physiol. 1978;278:471-490.

31. Morrell NW, Atochina EN, Morris KG, Danilov SM, Stenmark KR. Angiotensin converting enzyme expression is increased in small pulmonary arteries of rats with hypoxia-induced pulmonary hypertension. $J$ Clin Invest. 1995;96(4):1823-1833.

32. Guignabert C, de Man F, Lombes M. ACE2 as therapy for pulmonary arterial hypertension: the good outweighs the bad. Eur Respir J. 2018;51(6).

33. Tikellis C, Thomas MC. Angiotensin-Converting Enzyme 2 (ACE2) Is a Key Modulator of the Renin Angiotensin System in Health and Disease. Int J Pept.2012;2012:256294.

34. Hanafy S, Tavasoli M, Jamali F. Inflammation alters angiotensin converting enzymes (ACE and ACE-2) balance in rat heart. Inflammation. 2011;34(6):609-613.

35. Ferrario CM. ACE2: more of Ang-(1-7) or less Ang II? Curr Opin Nephrol Hypertens.2011;20(1):1-6.

36. Dilauro M, Zimpelmann J, Robertson SJ, Genest D, Burns KD. Effect of ACE2 and angiotensin-(1-7) in a mouse model of early chronic kidney disease. Am J Physiol Renal Physiol. 2010;298(6):F1523-1532.

37. Verdecchia P, Cavallini C, Spanevello A, Angeli F. The pivotal link between ACE2 deficiency and SARSCoV-2 infection. Eur J Intern Med. 2020;76:14-20.

38. Chappell MC. The Angiotensin-(1-7) Axis: Formation and Metabolism Pathways.Angiotensin-(1-7) 2019: $1-26$.

39. Eatman D, Wang M, Socci RR, Thierry-Palmer M, Emmett N, Bayorh MA. Gender differences in the attenuation of salt-induced hypertension by angiotensin (1-7).Peptides. 2001;22(6):927-933.

40. Almeida AP, Frabregas BC, Madureira MM, Santos RJ, Campagnole-Santos MJ, Santos RA. Angiotensin(1-7) potentiates the coronary vasodilatatory effect of bradykinin in the isolated rat heart. Braz J Med Biol Res.2000;33(6):709-713.

41. Bader M. ACE2, angiotensin-(1-7), and Mas: the other side of the coin. Pflugers Arch.2013;465(1):79-85.

42. Zhang C, Zhao YX, Zhang YH, Zhu L, Deng BP, Zhou ZL, Li SY, Lu XT, Song LL, Lei XM, et al. Angiotensin-converting enzyme 2 attenuates atherosclerotic lesions by targeting vascular cells. Proc Natl Acad Sci U S A.2010;107(36):15886-15891.

Figure legends

Figure 1.

The design of animal study. In the early treatment protocol, there were sham-operated rats, SU5416 combined with left pneumonectomy rats $\left(\mathrm{SuPNx}_{42}\right)$, and $\mathrm{SuPNx}_{42}$ rats given with $15 \mathrm{mg} \mathrm{DIZE} / \mathrm{kg} /$ day from day 1 
to day $42\left(\mathrm{SuPNx}_{42} / \mathrm{DIZE}_{1-42}\right)$. In the late treatment protocol, there were sham-operated rats (sham42), $\mathrm{SuPNx}_{42}$, and a SuPNx 42 rats given with $15 \mathrm{mg}$ DIZE $/ \mathrm{kg} /$ day from day 29 to day $42\left(\mathrm{SuPNx}_{42} / \mathrm{DIZE}_{29-42}\right)$

SuPNx: Su5416 with left pneumonectomy

DIZE: diminazene aceturate

Figure 2 Comparison of (A) right ventricle systolic pressure (RVSP), (B) Fulton index, the weight ratio of right ventricle to left ventricle plus septum, $(\mathrm{RV} / \mathrm{LV}+\mathrm{S}),(\mathrm{C})$ the lung weight and $(\mathrm{D})$ the body weight among the sham $42, \mathrm{SuPNx}_{42}, \mathrm{SuPNx}_{42} / \mathrm{DIZE}_{1-42}$ and $\mathrm{SuPNx}_{42} / \mathrm{DIZE}_{29-42}$ groups. RVSP levels were significantly higher in the $\mathrm{SuPNx}_{42}$ group $(45.17 \pm 4.62 \mathrm{mmHg})$ than in sham-operated rats. $(18.37 \pm 1.36 \mathrm{mmHg}, p$ $<0.001)$ Both DIZE treatment groups $\left(\mathrm{SuPNx}_{42} / \mathrm{DIZE}_{1-42}\right.$ and $\left.\mathrm{SuPNx}_{42} / \mathrm{DIZE}_{29-42}\right)$ showed significant decreases in RVSP when compared with that of the SuPNx 42 rats. (early, $25.10 \pm 1.38 \mathrm{mmHg}, p<0.01$; late $31.23 \pm 4.06 \mathrm{mmHg}, p<0.05)$ The Fulton index was significantly increased in the $\mathrm{SuPNx}_{42}$ group $(0.55 \pm$ $0.06)$ than in sham-operated group $(0.35 \pm 0.04, p<0.05)$, but no significant changes were observed in the $\mathrm{SuPNx}_{42} / \mathrm{DIZE}_{1-42}$ and $\mathrm{SuPNx}_{42} / \mathrm{DIZE}_{29-42}$ groups when compared with the $\mathrm{SuPNx}_{42}$ group. There was no significant change in either the lung or body weight in all groups. Values represent the mean \pm SEM. ${ }^{*} P$ $<0.05,{ }^{* *} P<0.01,{ }^{* * *} P<0.001$

Figure 3 (A) The plasma brain natriuretic peptide (BNP) and (B) RV BNP levels. The plasma BNP level was significantly higher in the $\mathrm{SuPNx}_{42}$ rats than in sham-operated rats. $(130.2 \pm 19.76$ vs. $59.50 \pm 7.74$ $\mathrm{pg} / \mathrm{ml} ; P<0.001)$ Treatment with DIZE for 42 days significantly lowered the plasma BNP levels when compared with that of the vehicle-treated group. $(31.14 \pm 2.83$ vs. $130.2 \pm 19.76 \mathrm{pg} / \mathrm{ml} ; P<0.05)$ There was a trend that the $\mathrm{SuPNx}_{42} / \mathrm{DIZE}_{29-42}$ rats had a decreased plasma BNP level when compared with the $\mathrm{SuPNx}_{42}$ rats, but the difference was not statistically significant. The RV BNP was significantly higher in $\mathrm{SuPNx}_{42}$ rats than that of the sham $_{42}$ rats. $(103.4 \pm 10.01$ vs. $31.05 \pm 2.57 \mathrm{pg} / \mathrm{ml} ; P<0.0001)$ Both DIZE treatment groups had significantly lowered RV BNP than in the $\mathrm{SuPNx}_{42}$ rats. (early: $46.82 \pm 6.62 \mathrm{pg} / \mathrm{ml}$ vs. $103.4 \pm 10.01 \mathrm{pg} / \mathrm{ml}, P<0.0001$; late: $61.83 \pm 5.10 \mathrm{pg} / \mathrm{ml}$ vs. $103.4 \pm 10.01 \mathrm{pg} / \mathrm{ml}, P<0.001)$ Values represent the mean \pm SEM. ${ }^{*} P<0.05 ;{ }^{* * *} P<0.001,{ }^{* * * *} P<0.0001$

Figure 4 The representative results of Hematoxylin-eosin stating of lung tissue (A), Verhoeff-van Gieson staining (B), and double immunohistochemical staining of smooth muscle actin (green) and PCNA (brown) (C). (D) The ratio of medial wall thickness of pulmonary arterioles. The value for the $\operatorname{sham}_{42}$ group was set at 1.0. The muscular layer of pulmonary arterioles (50-100 $\mu \mathrm{m}$ in diameter) was significantly thicker in the $\mathrm{SuPNx}_{42}$ rats than in $\operatorname{sham}_{42}, \mathrm{SuPNx}_{42} / \mathrm{DIZE}_{1-42}$, but not $\mathrm{SuPNx}_{42} / \mathrm{DIZE}_{29-42}$ rats. (E) the vascular occlusion score (VOS). The VOS of early treatment group $\left(\mathrm{SuPNx}_{42} / \mathrm{DIZE}_{1-42}\right)$ was decreased compared to $\mathrm{SuPNx}_{42}$. $(1.27 \pm 0.74$ vs. $1.07 \pm 0.56$ ) (magnification, 200x. Values represent the mean $\pm \mathrm{SEM} . * \mathrm{P}<0.05$, $* * \mathrm{P}<0.01$.

Figure 5 (A) A representative Western blot of eNOS, ACE, ACE2, and the MAS receptor in sham 42 , $\mathrm{SuPNx}_{42}, \mathrm{SuPNx}_{42} / \mathrm{DIZE}_{1-42}$ and $\mathrm{SuPNx}_{42} / \mathrm{DIZE}_{29-42}$ rats. (B) The normalized eNOS/actin ratio revealed the expression of eNOS was higher in the $\mathrm{SuPNx}_{42} / \mathrm{DIZE}_{1-42}$ rats than in $\mathrm{SuPNx}_{42}$ rats. (C) The normalized ratio of $\mathrm{ACE} /$ actin showed that the expression of $\mathrm{ACE}$ was higher in $\mathrm{SuPNx}_{42}$ rats than in $\mathrm{sham}_{42}$ rats. DIZE administration decreased ACE expression in the early treatment $\left(\mathrm{SuPNx}_{42} / \mathrm{DIZE}_{1-42}\right)$ and the late treatment $\left(\mathrm{SuPNX}_{42} / \mathrm{DIZE}_{29-42}\right)$ groups. (D) The normalized ratio of ACE2/actin revealed that an increase in the early treatment group $\left(\mathrm{SuPNX}_{42} / \mathrm{DIZE}_{1-42}\right)$ compared to the $\mathrm{SuPNx}_{42}$ rats. No significant differences were found among the sham $42, \mathrm{SuPNx}_{42}$, and $\mathrm{SuPNX}_{42} / \mathrm{DIZE}_{29-42}$ groups. (E) The normalized MAS/actin ratios were not changed in the all groups. The value for the $\operatorname{sham}_{42}$ group was set at 1.0 in Figures $5 \mathrm{~B}-5 \mathrm{E}$. Values represent the mean $\pm \mathrm{SEM} * P<0.05,{ }^{* *} P<0.01$

Figure 6 (A) The levels of RV Ang-(1-7). The level of RV Ang-(1-7) was significantly higher in the $\mathrm{SuPNx}_{42} / \mathrm{DIZE}_{1-42}$ and $\mathrm{SuPNx}_{42} / \mathrm{DIZE}_{29-42}$ rats than in $\mathrm{SuPNx}_{42}$ rats. (B) The levels of pulmonary Ang(1-7). The expression of pulmonary Ang-(1-7) was significantly higher in $\mathrm{SuPNx}_{42} / \mathrm{DIZE}_{1-42}$ rats than in $\mathrm{SuPNx}_{42}$ rats. Values represent the mean \pm SEM. ${ }^{*} P<0.05{ }^{* * *} \mathrm{P}<0.001,{ }^{* * * *} \mathrm{P}<0.0001$ 


\section{Figure S1}

The rat model of left pneumonectomy followed by SU5416 injection. Remodeling of neointimal occlusive lesions was observed in this animal model. It has plexiform-like lesions by allowing for the endothelial cell hyperproliferation, a distinct feature shown in human PAH.

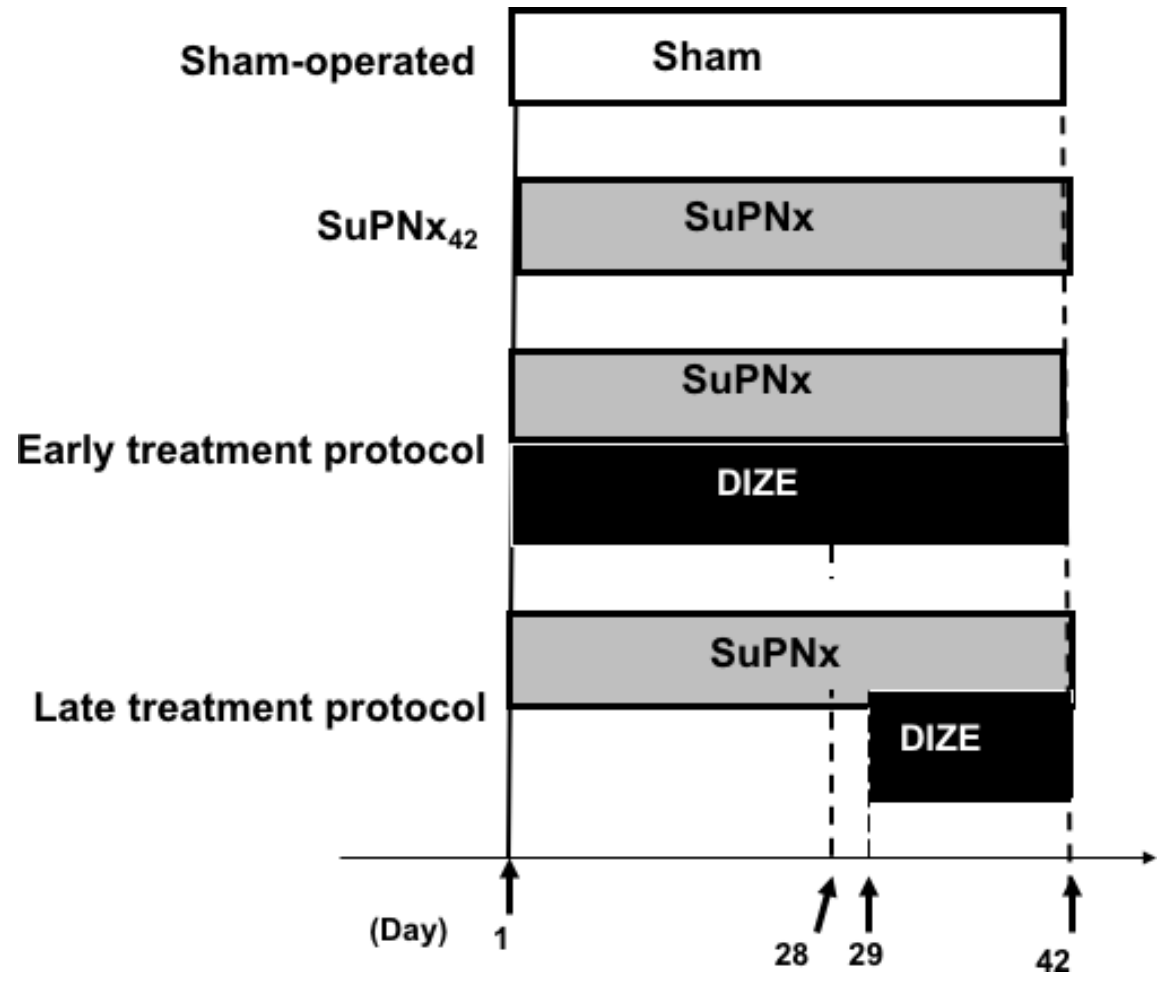

A

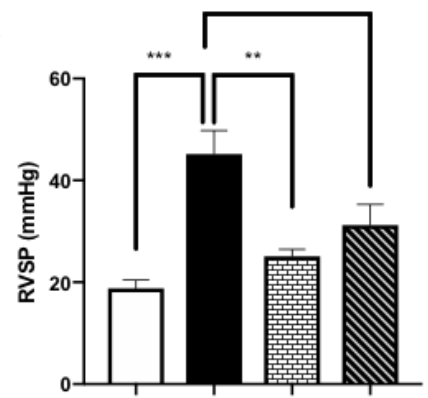

C

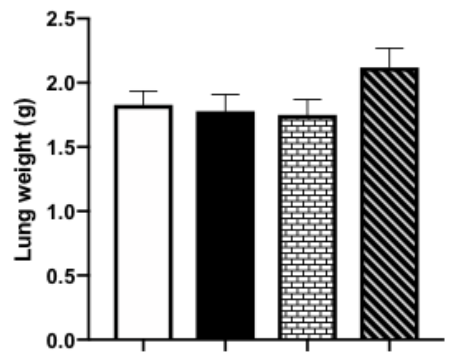

B

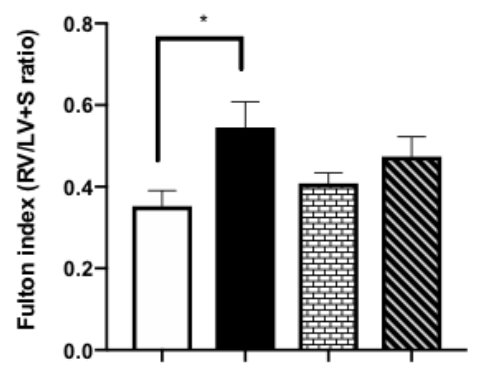

D

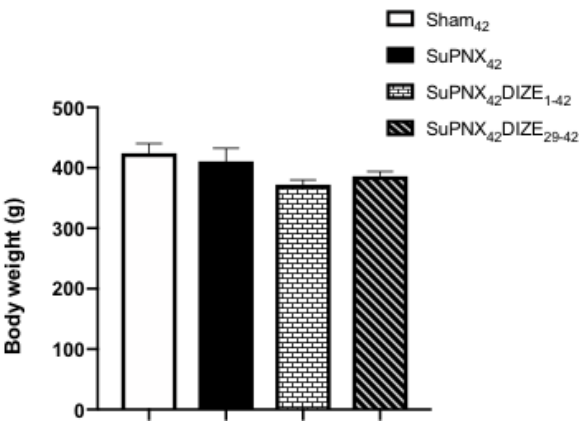



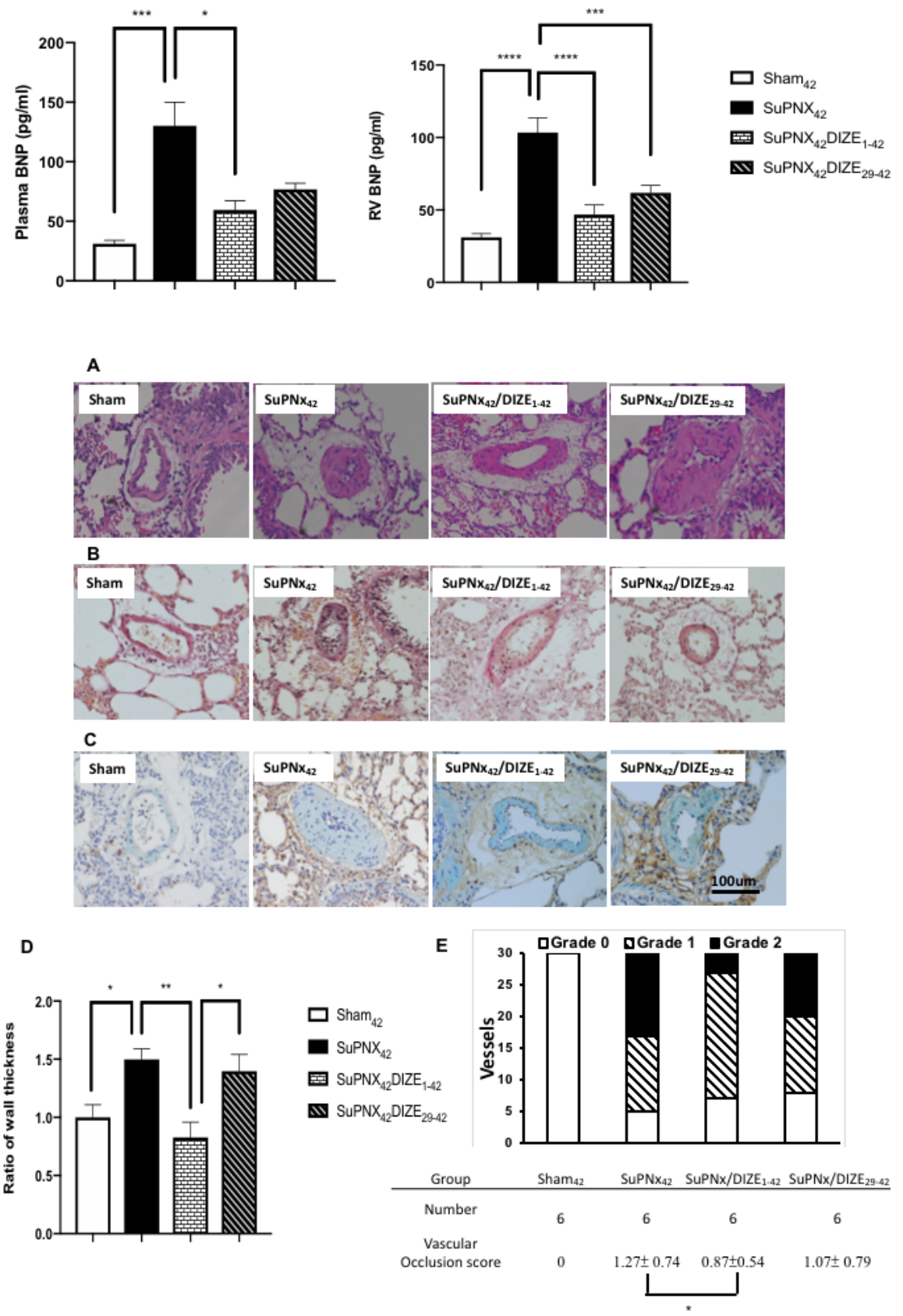

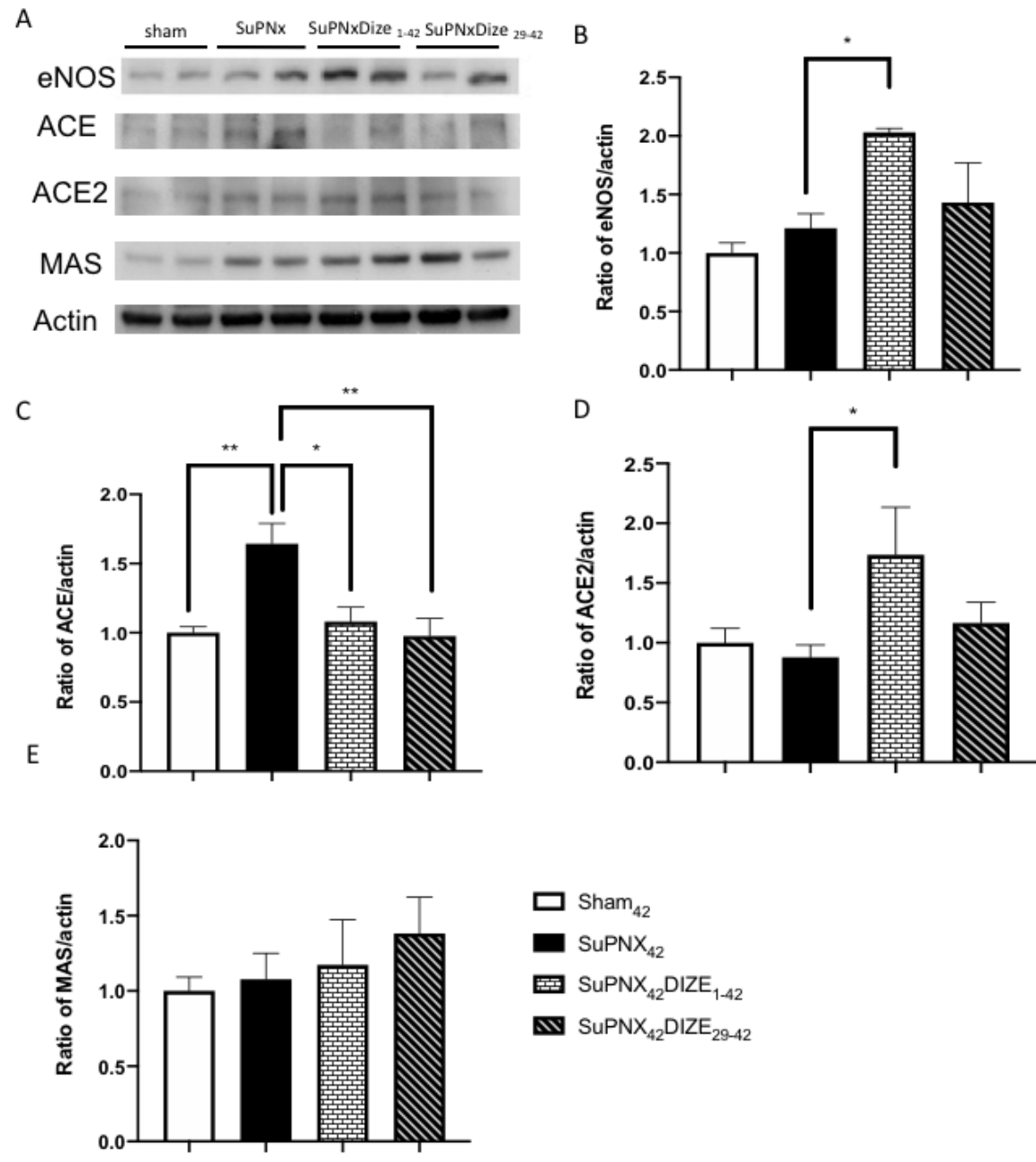

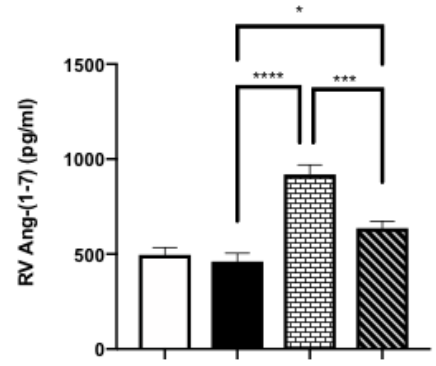

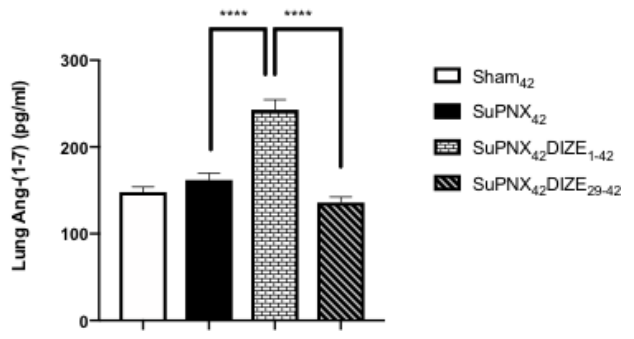




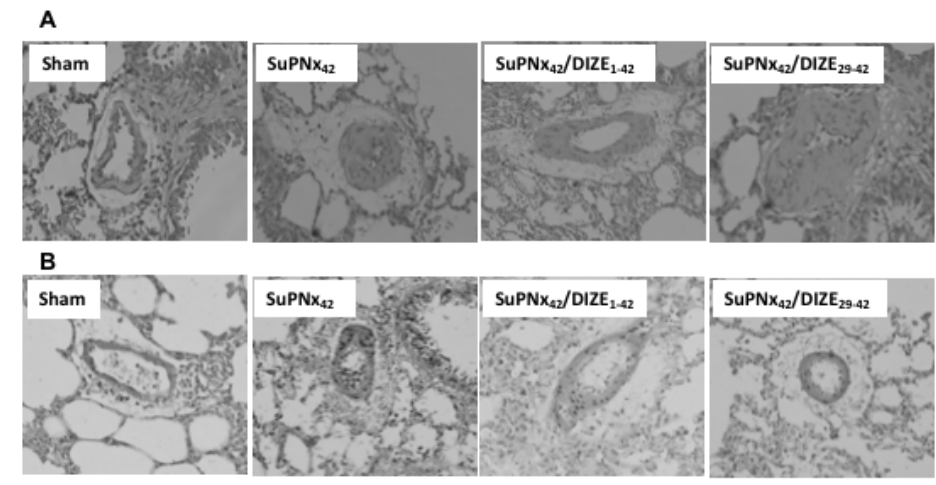

C

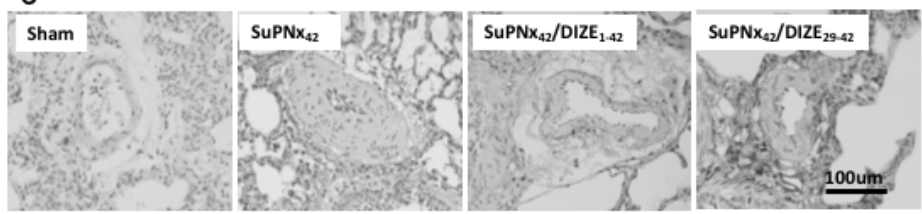

D

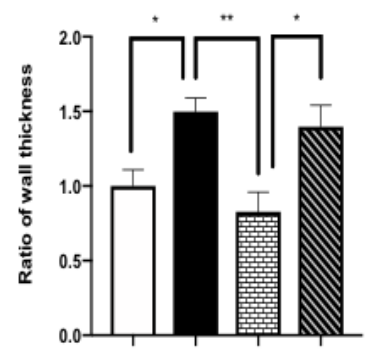

E

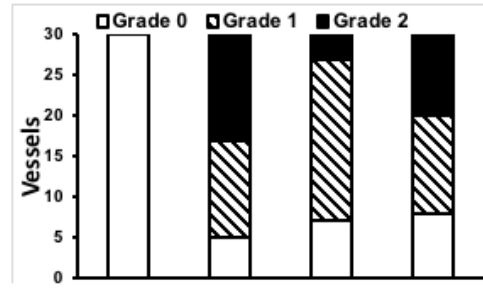

Group Sham

SuPNX $_{42}$ SUPNx/DIZE ${ }_{1-42}$ SuPNx/DIZE 29.42

Number
Vascular

Occlusion score

0
$1.07 \pm 0.79$ 